Please do not remove this page

RMIT

UNIVERSITY

\title{
Molecular dynamics simulation study of the static and dynamic properties of a model colloidal suspension with explicit solvent
}

Hannam, Stephen; Daivis, Peter; Bryant, Gary

https://researchrepository.rmit.edu.au/esploro/outputs/9921860338301341/filesAndLinks?institution=61RMIT_INST\&index=null

Hannam, S., Daivis, P., \& Bryant, G. (2016). Molecular dynamics simulation study of the static and dynamic properties of a model colloidal suspension with explicit solvent. Molecular Simulation, 42(6-7), 511-521. https://doi.org/10.1080/08927022.2015.1066505

Document Version: Accepted Manuscript

Published Version: https://doi.org/10.1080/08927022.2015.1066505

Repository homepage: https://researchrepository.rmit.edu.au

(C) 2015 Taylor and Francis

Downloaded On 2023/04/26 15:26:20 +1000

Please do not remove this page 
Thank you for downloading this document from the RMIT Research Repository.

The RMIT Research Repository is an open access database showcasing the research outputs of RMIT University researchers.

RMIT Research Repository: http://researchbank.rmit.edu.aul

\author{
Citation: \\ Hannam, S, Daivis, P and Bryant, G 2016, 'Molecular dynamics simulation study of \\ the static and dynamic properties of a model colloidal suspension with explicit \\ solvent', Molecular Simulation, vol. 42, no. 6-7, pp. 511-521.
}

See this record in the RMIT Research Repository at:

https://researchbank.rmit.edu.au/view/rmit:36449

Version: Accepted Manuscript

Copyright Statement:

(C) 2015 Taylor and Francis

Link to Published Version:

http://dx.doi.org/10.1080/08927022.2015.1066505 
Please cite as:

Hannam, S.D.W., Daivis, P.J., Bryant, G.

"Molecular dynamics simulation study of the static and dynamic properties of a model colloidal suspension with explicit solvent," Molecular Simulation 42, 511-521 (2016). 
Vol. 00, No. 00, Month 200x, 1-15

\title{
RESEARCH ARTICLE
}

\section{Molecular dynamics simulation study of a model colloidal suspension}

\author{
S.D.W. Hannam, ${ }^{\text {a }}$ P.J. Daivis ${ }^{\mathrm{a} *}$ and G. Bryant ${ }^{\mathrm{a}}$ \\ ${ }^{a}$ School of Applied Sciences, RMIT University, GPO Box 2476, Melbourne Vic 3001, Australia;
}

$(x x x)$

\begin{abstract}
Molecular dynamics simulation was used to study a colloidal suspension with explicit solvent to determine how inclusion of the solvent affects the structure and dynamics of the system. The solute was modelled as a hard-core particle enclosed in a WCA potential shell, while the solvent was modelled as a simple WCA fluid. We found that when the solute-solvent interaction included a hard core equal to half of the solute hard core diameter, large depletion effects arose, leading to an effective attraction and large deviations from hard-sphere structure for the colloidal component. It was found that these effects could be eliminated by reducing the hard-core distance parameter in the solute-solvent interaction, thus allowing the solvent to penetrate closer to the colloidal particles. Three different values for the solute-solvent hard-core parameter were systematically studied by comparing the static structure factor and radial distribution function to the predictions of the Percus-Yevick theory for hard-spheres. When the optimal value of the solute-solvent hard core interaction parameter was found, this model was then used to study the dynamical behaviour of the colloidal suspension. This was done by first measuring the velocity autocorrelation function over a large range of packing fractions. We found that this model predicted the sign of the long time tail in the velocity autocorrelation function in agreement with experimental values, something that single component hard-sphere systems have failed to do. The intermediate scattering functions at low wavevector were briefly studied to determine their behaviour in a dilute system. It was found that they could be modelled using a simple diffusion equation with a wavevector independent diffusion coefficient, making this model an excellent analogue of experimentally studied hard sphere colloids.
\end{abstract}

Keywords: Molecular dynamics, Colloid, Hard sphere, Explicit solvent, Intermediate scattering function

\section{Introduction}

Colloidal suspensions provide an ideal system for studying phase transitions as their particle size and diffusive dynamics ensure that crystallisation generally occurs slowly enough for it to be studied experimentally in real time. In the past, the best computational analogue to a real colloidal system was a single component hard-sphere (HS) fluid, which is usually simulated using event driven molecular dynamics.[1] In these single component HS fluid simulations, the presence of the solvent is completely ignored. Instead, the HS particles move ballistically (feeling no forces from surrounding particles, until they encounter another HS particle), at which time they undergo an elastic collision. This is quite different from the behaviour of real colloidal particles which interact directly (through interparticle interactions), and indirectly by momentum transfer via the solvent (hydrodynamic interactions).[2] This second type of interaction causes the colloidal particles to diffuse through the solvent, moving at a much slower rate than a single component HS particle without solvent. However, even though the single component HS fluid has dynamics that are very different from real colloidal suspensions, many of the structural properties and phase behaviour are consistent.

*Corresponding author. Email: peter.daivis@rmit.edu.au 
HS crystallisation was first demonstrated experimentally in 1986, when Pusey and van Megen [3] systematically studied the behaviour of suspensions of colloidal particles in a solvent at various packing fractions ${ }^{1}$. The authors found that by increasing the concentration the samples would progress from a fluid, to fluid and crystal coexisting, to fully crystallised samples. By mapping the phase behaviour onto the HS system, it was shown that the packing fractions where a phase change occurs agrees with that of the theoretical HS system. For an ideal single component HS system, it remains a fluid up to a packing fraction of 0.495 , then between 0.495 and 0.545 there is fluid-crystal coexistence and above 0.545 the sample is crystalline.[5]

In addition to the phase behaviour matching the HS system, the local structure of the colloidal suspension also matches closely. Several techniques such as small angle scattering of light,[6] Xrays [7] and neutrons [8] have been used to probe the static structure factor of suspensions. These have been compared to Percus-Yevick theory for hard-spheres [9] and good agreement has been found in the fluid region.[10]

However, even with all the success of the HS model in matching the structural properties and phase behaviour, there are some dynamical properties that do not agree completely. For example, there is currently a discrepancy between the rate of crystallisation; where the nucleation rate densities obtained by computer simulation differ significantly from those observed in experiments.[11-15] This is thought by some authors to be due to the fact that the hydrodynamic interactions (HIs) are not taken into account, and attempts have been made to include HIs to resolve the discrepancy.[16, 17] These authors found that including HIs in the model drastically changes the rate of crystallisation, but the disagreement remains.

The velocity autocorrelation function has also been calculated in great detail for the onecomponent HS model in simulations over a wide range of packing fractions [18] and shows similarities to experimental results. Simulations and experiments both show the classic $-3 / 2$ power law decay at long delay times, but the simulations found that this decay was always from above zero for packing fractions lower than 0.45 , while experimental results show that it always decays from below zero, except for extremely dilute samples.[19-21] This discrepancy could be caused by neglecting effects due to the solvent, such as its viscoelasticity or solvent-mediated hydrodynamic momentum transfer. Therefore, it is of interest to see whether inclusion of the solvent gives a better match to real colloidal systems.

Simulations of colloidal suspensions are often done using Langevin/Brownian dynamics where inclusion of HIs can be quite difficult. This is because in a dilute system, the force on each Brownian particle from the solvent is independent and has the form given by Stokes' law. Whereas for moderate densities, the force on each Brownian particle from the solvent depends on the motion of all other Brownian particles. These many-body interactions can be complicated and difficult to implement, $[22,23]$ which makes the technique of simulating the solvent particles explicitly an attractive method as this only involves the calculation of two-body interactions.

Very few attempts have been made to include solvent explicitly into the simulation by calculating the equations of motion for both the colloidal particles and the solvent directly. This is simply because the computational cost is far too high to have solute to solvent size and mass ratios that are even close to the experimental conditions. Therefore, any attempts made so far have used a mass ratio that is much smaller than the experimental mass ratio, but still big enough that the behaviour of the heavier colloidal particles is still governed by Brownian dynamics. [4, 24]

One of the few attempts was made by Vrabecz and Toth [25] who studied the effect of explicitly adding a second smaller HS particle (1/5th the diameter of the larger colloidal particle) on the structural properties of the fluid. They found that including the second smaller species caused a change in the structure of the colloidal particles in the fluid. This was evident through sharpening in the peaks of the radial distribution function, showing the presence of the smaller particles

\footnotetext{
${ }^{1}$ In the experimental systems, the volume fraction and the packing fraction are the same because the solvent molecules are infinitesimally small. However in the simulations with a finite solute to solvent size ratio these two parameters are no longer equivalent due to unoccupied space between the particles. In other words the thermodynamic volume fraction of a single component fluid is always 1 , while the packing fraction is not [4].
} 
causes an effective attraction between the colloidal particles.

The sharpening of the peaks in the radial distribution function is most likely due to depletion effects caused by an overlap of the excluded volume of colloidal particles as they approach one another [26]. The inability of the solvent to fit between the colloidal particles means that the pressure surrounding them is greater than the pressure between them. The colloidal particles therefore feel an effective attraction, known as a depletion attraction. For a real colloidal suspension, depletion effects also exist but they are negligible as the solvent particles are infinitesimally small. As the number of particles we can simulate is limited, we must use a finite solute/solvent size ratio, so we need to develop simulation techniques to reduce the depletion effects.

Therefore, the goal of this paper is to construct a two component model of colloidal particles + solvent that will have structural properties which match with both experiment and theory for a HS system, but in which the colloidal particles interact with an explicit solvent making their dynamics diffusive, rather than ballistic. This will involve finding a way to reduce the depletion effects that can dominate the system when this second species is added. Once this model is found to match the structural properties, we will study the phase behaviour and dynamical properties to see if the behaviour matches the experimental/theoretical systems.

The remainder of the paper is organised as follows. We will first introduce the pair potential used to model the interactions in the system, and the method for estimating the packing fraction. Then, we compare the radial distribution function and static structure factor to experimental/theoretical data for three systems using different values of the solute-solvent hard-core parameter. Once this parameter is optimised, the phase behaviour and crystal structure will be compared with theory/experiment. Finally, some dynamical properties such as the velocity autocorrelation function and intermediate scattering function will be measured to see how the introduction of the explicit solvent effects the dynamical properties when compared to a single component HS system.

\section{Simulation Method}

\subsection{Simulation Pair Potentials}

The parameters for our simulations were chosen with the goal of creating a model which represents large colloidal particles (also referred to in paper as solute) dispersed in a fluid with a large number of much smaller solvent particles. To do this we modelled the colloidal particle and solvent using a Weeks-Chandler-Andersen (WCA) potential (a shifted and truncated LennardJones potential) which is modified to include a hard core.[27] The WCA potential takes the form

$$
\phi\left(r_{i j}\right)= \begin{cases}\infty & \text { if } r_{i j} \leq c_{a b} \\ 4 \epsilon\left[\left(\frac{\sigma}{r_{i j}-c_{a b}}\right)^{12}-\left(\frac{\sigma}{r_{i j}-c_{a b}}\right)^{6}\right]+\epsilon & \text { if } c_{a b}<r_{i j}<c_{a b}+2^{1 / 6} \\ & \text { otherwise }\end{cases}
$$

where $r_{i j}$ is the centre-to-centre distance between the particles $i$ and $j, \epsilon$ is the depth of the potential well, $\sigma$ is the nominal length scale of the potential (in this work $\epsilon=\sigma=1$ ). The $c_{a b}$ parameter introduces a hard-core to the potential where $a$ and $b$ are the two interacting species. This creates an excluded region which is used to increase the size of the colloidal particle relative to the solvent. For convenience, we will consider the colloidal particles as species 1 and the solvent as species 2 .

In this work we will keep the value of the $c_{a b}$ fixed for the solute-solute $c_{11}$ and solventsolvent $c_{22}$ interaction at 3.034 and 0.000 respectively. These values give the colloidal particles an effective diameter of 4.034 times the diameter of the solvent particles (the way this is calculated is explained in the next section). 
The $c_{12}$ parameter for the solute-solvent interaction will be varied. We will compare three different values denoted as systems A, B and C. This allows us to keep the strength of the interactions constant, while the excluded region around the colloidal particles that the solvent cannot access is varied. The values of $c_{12}$ for systems A, B and C are 1.515, 1.015 and 0.000 respectively.

We have chosen these three particular values because they cover three distinct situations. System A corresponds to the usual mixing rule, $c_{12}=\left(c_{11}+c_{22}\right) / 2$. This results in an excluded region around the colloidal particles that the centre of mass of the solvent cannot access (as in a usual binary HS fluid). Assuming that the effective hard sphere diameter of the WCA solvent (calculated using the method discussed later) is equal to 1.0, this allows the solvent and solute to effectively touch at their hard sphere radii. System B reduces the value of $c_{12}$ to allow the centre of mass of the solvent to reach the outside of the colloidal particles, and therefore eliminate the excluded region. System C eliminates the hard core in the solute-solvent interactions completely, and so allows the solvent centre of mass to penetrate the colloidal particles.

\section{$2.2 \quad$ Simulation Conditions}

All simulations were performed using the MD package LAMMPS [28] and post-processed using in-house code. Simulations at each packing fraction were done under NPT conditions at a reduced temperature of 1.00 and reduced pressure of 7.85 with a time step of 0.005 . The temperature is held fixed using a Nose-Hoover thermostat and the pressure is held fixed using a Nose-Hoover type barostat, both used a damping parameter of 10 . This was done in order to better replicate the experimental conditions of a real colloidal suspension, and by having the pressure the same at different packing fractions it guarantees the state point of the solvent is constant as the packing fraction is increased. All simulations were done with a total of 32000 particles.

\subsection{Calculation of effective diameter and packing fraction}

The packing fraction of a species is equal to the total volume taken up by all the atom of the species, divided by the total volume of the system. In order to calculate this quantity, the typical volume of an atom of that species needs to be known. In usual hard-sphere simulations the diameter of the particle is clear, but in this work we are using a hard-core plus a WCA repulsive potential. This WCA repulsive potential adds an extra diameter that is not clearly defined.

Previous work has been done by Hess et. al. to determine expressions for the effective HS diameter of WCA particles as a function of temperature by comparing thermodynamic properties with those predicted by the Carnahan-Starling equation of state for the HS fluid.[29] These authors found that the method which gave the best agreement with the MD results was to define the effective HS diameter $d$ to be the interparticle separation at which the interaction potential is equal to Boltzmann's constant times the temperature $\left(\phi(d)=k_{B} T\right)$. At the reduced temperature of 1.00 used in this work, this gives an extra diameter of 1.00 to the particles due to the WCA repulsion. Therefore, the colloidal particles in this work have an effective diameter of $d_{1}=3.034+1.000=4.034$ while the solvent has an effective diameter of $d_{2}=0.000+1.000=$ 1.000 .

As with a real colloidal suspension, our packing fraction is calculated purely from the volume taken up by the colloidal particles. Since the volume for each colloidal particle is $V_{c}=d_{1}^{3} \pi / 6=$ $4.034^{3} \pi / 6$, the packing fraction $\eta$ can be estimated as $\eta=4.034^{3} \pi N_{c} / 6 V$ where $N_{c}$ is the total number of colloidal particles and $V$ is the total volume of the system. Comparing the phase behaviour of this system to that of a hard-sphere system will show us whether this estimate of the packing fractions is accurate.

The mass of the colloidal particle was set with the goal of making it approximately neutrally buoyant in the solvent. The mass needed to do this was calculated in the same way as McPhie 
[30], which for a size ratio $d_{1} / d_{2}$ of 4.034 gives a mass ratio $m_{1} / m_{2}$ of 50 . This size and mass ratio has been shown to be large enough for the larger particle to behave as a Brownian particle in the solvent. $[4,24]$

\subsection{Calculation of properties}

To compare our simulations to experiment/theory we will calculate a number of structural and dynamical properties. The structural order between the particles in the system can be quantified by the radial distribution function $g_{a b}(r)$ which gives the probability (relative to random) of finding a particle of type $a$ at a distance $r$ from another given particle of type $b$. This is a useful property to analyse, but it cannot be measured directly in an experiment. Instead, a more useful quantity to compare is the colloidal particles static structure factor $S(k)$ that can be measured in scattering experiments. [31, 32] The $S(k)$ can be computed from the $g_{11}(r)$, but a more convenient way is to compute it directly from its definition given as

$$
S(k)=\frac{1}{N}\left\langle n(\boldsymbol{k}, 0) n^{*}(\boldsymbol{k}, 0)\right\rangle
$$

where $\boldsymbol{k}$ is the wavevector being investigated, $N$ is the number of colloidal particles and

$$
n(\boldsymbol{k}, t)=\sum_{i=1}^{N} \exp \left(-i \boldsymbol{k} \cdot \boldsymbol{r}_{i}(t)\right)
$$

is the Fourier transform of the microscopic number density at time $t$. Since the simulations are done under periodic boundary conditions, the density is periodic in the box length $L$. Therefore $S(k)$ can only be computed at discrete values of $k$ given as $\boldsymbol{k}=\frac{2 \pi}{L}\left(n_{x}, n_{y}, n_{z}\right)$ where $n_{x}, n_{y}$ and $n_{z}$ are integers ${ }^{1}$. In this work, $S(k)$ only depends on the magnitude of $k$ as it has been averaging over all $\boldsymbol{k}$ of equal magnitude. For an isotropic fluid this is no problem as the results in each direction should be the same (on average). But for the solid phase, different directions will give different values depending on their orientation with respect to the lattice planes. Therefore, by averaging over all $k$ of equal magnitude, we are averaging over different crystal orientations, which is similar to what is done in power diffraction experiments. But, it is important to note that we are limited to only being able to average over directions consistent with the periodic boundary conditions for each magnitude of k. Therefore, the magnitude of our crystal $S(k)$ may not agree quantitatively with experiment, but it should allow us to find the wavevectors corresponding to scattering peaks.

The $S(k)$ measures density-density correlations at the same point in time, but this can be generalised to measure correlations in the density at two different points in time. This is done through the intermediate scattering function $F(k, t)$ given as

$$
F(k, t)=\frac{\left\langle n(\boldsymbol{k}, t) n^{*}(\boldsymbol{k}, 0)\right\rangle}{\left\langle n(\boldsymbol{k}, 0) n^{*}(\boldsymbol{k}, 0)\right\rangle}
$$

which also only depends on magnitude $k$. This property forms the basis of a wide range of scattering experiments that can provide different types of information, depending on the number of components, their sizes and interactions, and their scattering amplitudes. It has been studied extensively in experiment [33] and is often used in the study of glass transitions as the behaviour of $F(k, t)$ shows clearly the presence of structural arrest. [34]

The last property we studied was the velocity autocorrelation function of the colloidal particles $C(t)$ which measures the correlation in the velocity of the colloidal particles over time, and gives

\footnotetext{
${ }^{1}$ This condition is obtained by expanding any space dependent quantity as a Fourier series with periodicity equal to L
} 
a measure of how velocity fluctuations decay. The expression for the velocity autocorrelation function is

$$
C(t)=\frac{1}{3}\langle\boldsymbol{v}(t) \cdot \boldsymbol{v}(0)\rangle
$$

where $\boldsymbol{v}(t)$ is the velocity of the tagged colloidal particle at time $t$. We measured $C(t)$ for the colloidal particles as we want to compare our results with previous experimental and simulation results.

\section{Results and Discussion}

\subsection{Liquid static structure factor}

The colloidal particle static structure factor $S\left(k d_{1}\right)$ measured in real colloidal systems has been shown to match very closely with the Percus-Yevick (PY) theory for hard spheres in the fluid region. [10, 35] Therefore, a comparison between the results from simulation and the predictions from this theory is a good test as to whether the system structure matches the expected experimental behaviour. $S\left(k d_{1}\right)$ was calculated for systems A, B and C at approximately the same packing fractions. These are shown in Fig. 1 (dots) along with the predictions from PY theory (lines).

The position and magnitude of the peaks in system A disagree with PY theory. This indicates that the solvent strongly effects the structure of the colloidal particles, forcing them into a state they would not be in if the solvent were not present. The peak position of $S(k)$ corresponds to the wavelength of the average inter-particle separation in a fluid. Since $S\left(k d_{1}\right)$ for system A has a peak at higher $k$ than is predicted from PY theory, this indicates a smaller particle separation. This is most likely due to strong depletion forces in this system, as we will see later when we examine $g_{11}\left(r / d_{1}\right)$.

System B shows a much better match to the predictions. Since we are allowing the solvent to reach the outside of the colloidal particle, the excluded volume and thus depletion effects have been greatly reduced. The peak in $S\left(k d_{1}\right)$ now occurs much closer to the predicted $k$ value, indicating the inter-particle separation is closer to the expected value. This peak value still differs slightly from the prediction, and it can be seen that at higher $k$ the deviations of the measured $S\left(k d_{1}\right)$ from PY theory increase.

Decreasing the core interaction parameter to zero, as we have done in system $\mathrm{C}$, brings the simulation results and the PY theory predictions into almost perfect agreement, even at large $\mathrm{k}$ values. This indicates that the structure of the fluid matches very closely to an ideal HS fluid. The $S\left(k d_{1}\right)$ for systems B and $\mathrm{C}$ are very close, but the difference between the structures of these systems is seen more clearly in the $g_{11}\left(r / d_{1}\right)$ shown in the next section.

\subsection{Liquid radial distribution function}

A plot of the colloidal particle radial distribution functions $g_{11}\left(r / d_{1}\right)$ for the three systems at various packing fractions is shown in Fig. 2. All systems have their first peak at $d_{1}$ as expected, but the shape of the peaks is very different between systems. System A has a very sharp first peak that does not increase as the packing fraction increases. This is followed by a decrease almost to zero at approximately $1.25 d_{1}$. This decrease in the probability of finding two colloidal particles at a separation of $1.25 d_{1}$ indicates that particles that are at this separation feel a strong attraction. This is an interesting observation, because at a separation of $1.25 d_{1}$, the excluded volumes of the two colloidal particles start to overlap (and strong depletion forces begin to act). This is further evidence that the attraction is caused by depletion effects, in agreement with Vrabecz and Toth.[25] 

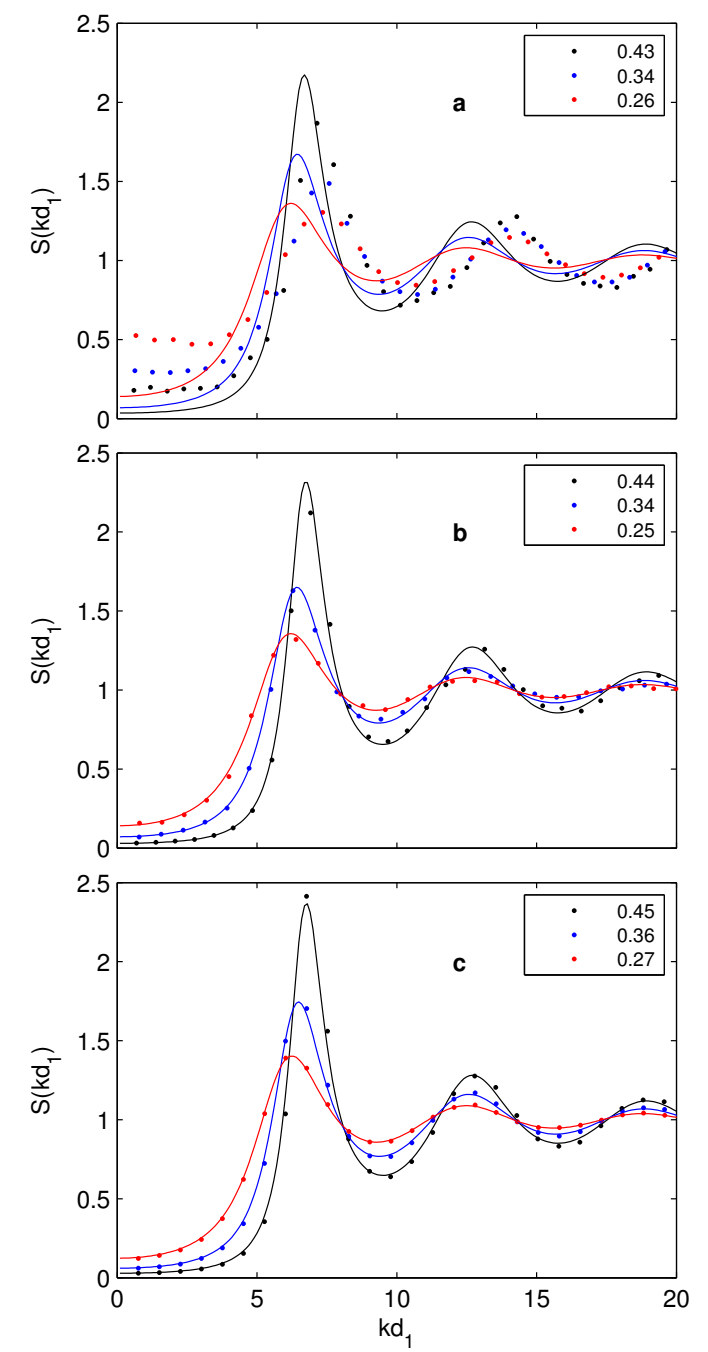

Figure 1. Plot of the colloidal particles static structure factor $S\left(k d_{1}\right)$ measured in simulation (dots) and from PY theory (lines) at a range of $\mathrm{k}$ vectors for packing fractions in the fluid state. a) Using usual mixing rules $\left(c_{12}=1.515\right)$, $\left.\mathrm{b}\right)$ using a smaller core value which allows the solvent to touch the outside of the colloidal particle $\left(c_{12}=1.015\right)$ and $\left.\mathrm{c}\right)$ no core parameter $\left(c_{12}=0.000\right)$.

The plot of System B shows that the depletion effects have been greatly reduced, but there is still a very sharp first peak followed by a minor secondary peak. In between these first two peaks, we still see a region where there is a decrease in the probability of finding a colloidal particle, indicating there is still some attractive force when they reach this separation. This may be because even though we have reduced the hard-core parameter to allow the solvent to fit between two colloidal particles, it is still energetically unfavourable for it to do so, especially when the colloidal particles are only 4 times their size. If the size ratio were much larger, as it is in real colloidal fluids, we would not expect this to occur.

It is only when we reduce the hard-core parameter to zero, and make the solute-solvent potential a pure WCA without any hard-core component, that the solvent can now freely move in between the colloidal particles. The $g_{11}\left(r / d_{1}\right)$ of system $\mathrm{C}$ now matches what we expect for a single component HS fluid, even though hydrodynamic effects are still present.

A comparison has also been made between the solute-solvent $g_{12}\left(r / d_{1}\right)$ and solvent-solvent $g_{22}\left(r / d_{1}\right)$ radial distribution functions. Fig. 3 shows a plot of $g_{12}\left(r / d_{1}\right)$ and $g_{22}\left(r / d_{1}\right)$ at a packing fraction of 0.36 . The first peak position of $g_{12}\left(r / d_{1}\right)$ in Fig. 3a clearly shows the differences between these 3 systems. By comparing the peak positions to the value of $0.5 d_{1}$ (which represents the outside radius of a colloidal particle) we can see how close the solvent is able to get to a 

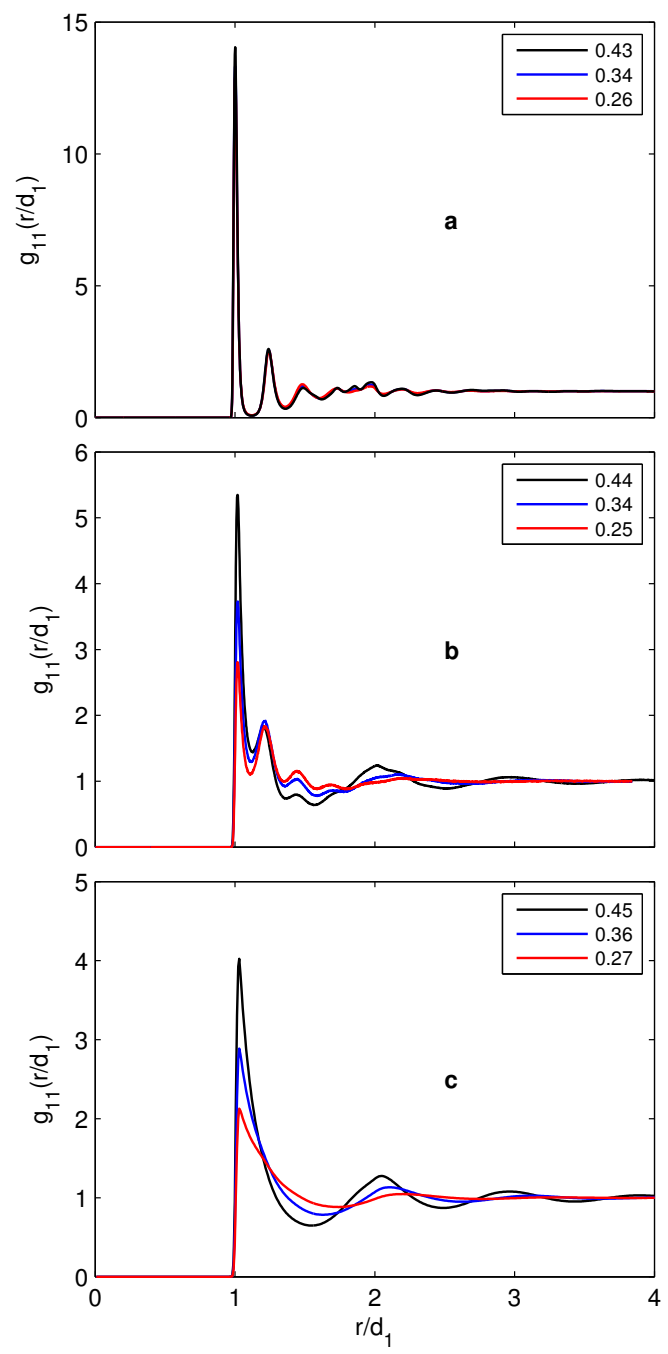

Figure 2. Plot of the colloidal particle radial distribution function $g_{11}\left(r / d_{1}\right)$ for a) Using usual mixing rules $\left(c_{12}=1.515\right)$, b) using a smaller core value which allows the solvent to touch the outside of the colloidal particle $\left(c_{12}=1.015\right)$ and $\left.\mathrm{c}\right)$ no core parameter $\left(c_{12}=0.000\right)$.

colloidal particle in all 3 systems. System A has its first peak at $r d_{1}>0.5$, showing the excluded region between $0.5<r d_{1}<0.75$ that the solvent does not have access to. System B has had that region removed, which has greatly reduced the depletion effects, while system $\mathrm{C}$ shows the solvent is able to penetrate into the colloidal particles.

Fig. 3b shows $g_{22}\left(r / d_{1}\right)$ for each of the systems at a packing fraction of 0.36 . They all have a peak at the same position, but the magnitudes of the peaks differ. This is because by using a smaller HS core value, we are giving the solvent access to more volume (even at constant pressure), which decreases its density.

In summary, even though system $\mathrm{C}$ uses a technique that could be considered un-physical since it allows the solvent to penetrate the colloidal particles, it does solve a problem that is inherent in these types of simulations where the size ratio is much smaller than found in experiments. Using a hard-core parameter of zero, and so making the solute and solvent interact with a pure WCA potential, results in a fluid that has the structural properties that match experiment and theory. Therefore, for the rest of the paper we will use this value of the hard-core parameter in the solute-solvent pair interactions. 

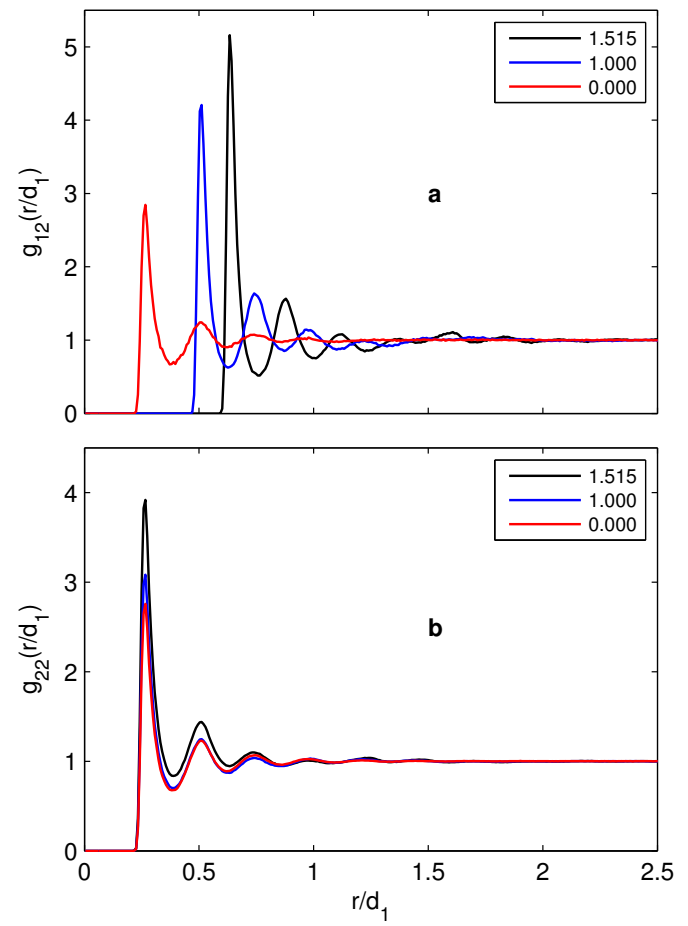

Figure 3. Plot of the radial distribution function at a packing fraction of 0.36 for a) the solute-solvent interaction $g_{12}\left(r / d_{1}\right)$ and $\mathrm{b}$ ) the solvent-solvent interaction $g_{22}\left(r / d_{1}\right)$, for the three values of $c_{a b}$ given in the legend.

\subsection{Phase behaviour}

Since system $\mathrm{C}$ has the fluid structure we desire, we move on now to study the phase behaviour and crystal structure. For the experimental colloidal suspension, the phase behaviour also follows the hard-sphere system very well. $[3,36]$ Fig. 4 shows a plot of the $g_{11}\left(r / d_{1}\right)$ at packing fractions just below freezing $(\eta=0.49)$, in the coexistence region $(\eta=0.53)$ and in the crystal region $(\eta=0.57)$. At the packing fraction just below freezing, $g_{11}\left(r / d_{1}\right)$ shows a liquid structure. However, as the packing fraction increases above the freezing point, structure starts to emerge at $r>r_{\text {peak }}$ which is characteristic of crystal planes forming. This is seen better in the $S\left(k d_{1}\right)$ shown in Fig. 5 for the same packing fractions. In the liquid region we see the usual form that can be predicted from PY theory, but as the packing fraction is increased into the coexistence region, the main peak in the structure factor greatly increases as the colloidal particles begin to re-arrange and crystallise. When we get into the solid region, peaks at larger $k$ start to develop indicating the emergence of lattice planes in the crystal.

In a real light scattering experiment, calculating the powder diffraction pattern requires orientational averaging of the crystal. In these simulations, the number of directions we can average over for each wave vector $k$ is limited. Because of this, when calculating the $S(k)$ for the coexistence and crystal region we have created a histogram of wavevectors with a bin width of $0.2 k d_{1}$ and averaged over the measurements in each bin. This allows us to average over more directions, however it decreases the resolution of our $S(k)$. Therefore, the magnitude of the peaks in the crystal $S\left(k d_{1}\right)$ may not agree quantitatively with experiment in the crystal region, but the position of the peaks should be comparable to experiment. Iacopini et. al. have measured the crystal structure factor for hard-sphere-like polystyrene microgel colloids dispersed in solvent using time resolved light scattering [31]. These authors found the $k$ values corresponding to the main peaks in the crystal structure factor were approximately $8.5 \mu \mathrm{m}^{-1}, 13.8 \mu \mathrm{m}^{-1}$ and $16.3 \mathrm{\mu m}^{-1}$. Multiplying these by their estimate for the colloid diameter of $846 \mathrm{~nm}$ gives $7.2,11.7$ and 13.8 as approximate values of $k d_{1}$ where peaks should occur. These values are also shown in Fig. 5 (arrows). 


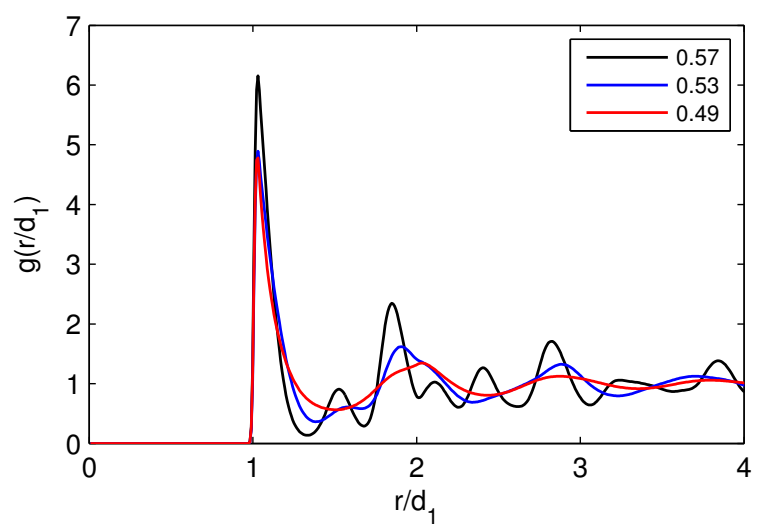

Figure 4. Plot of the colloidal particle radial distribution function $g_{11}\left(r / d_{1}\right)$ at packing fractions just below freezing $(\eta=0.49)$, in the coexistence region $(\eta=0.53)$ and in the solid region $(\eta=0.57)$.


Figure 5. Plot of the colloid-colloid static structure factor $S\left(k d_{1}\right)$ measured in simulation at packing fractions just below freezing $(\eta=0.49)$, in the coexistence region $(\eta=0.53)$ and in the solid region $(\eta=0.57)$. Vertical lines correspond to position of Bragg peaks as found from experiment [31]. 


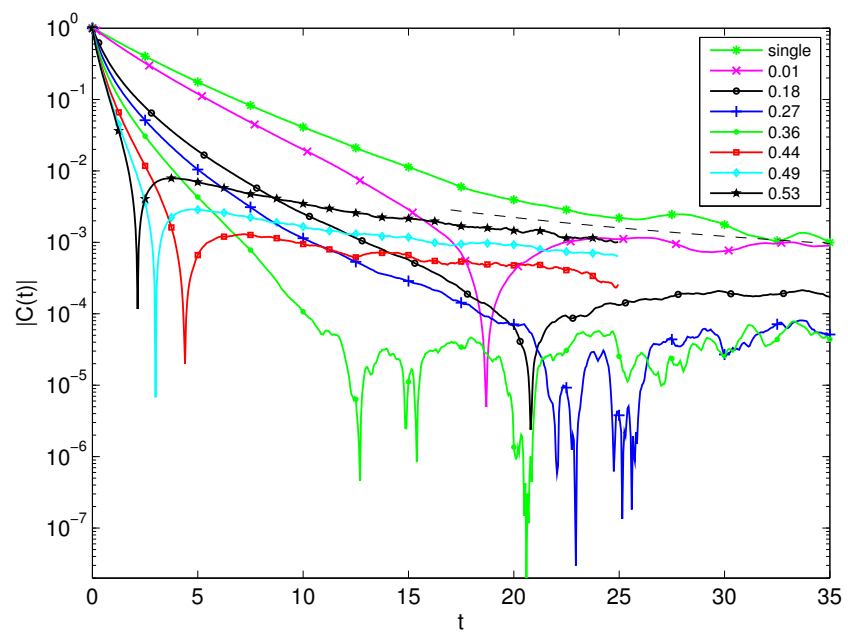

Figure 6. Log-linear plot of the velocity autocorrelation function measured in simulation at a range of packing fractions along with a $t^{-3 / 2}$ power law decay (dotted line).

A comparison between the peak positions of our results to the experimental peaks shows quite good agreement, indicating the structure of the crystal formed in our simulation matches closely to the crystal formed in experiment. In the analysis done by Iacopini et. al. they note that the observed scattering peaks correspond to the FCC 111, 220, 311 and HCP 002, 110, 112 planes. Therefore, the scattering pattern can be interpreted in terms of random hexagonal close packed,[31] showing that hard spheres crystallise by stacking hexagonal closed packed planes. We do not see the HCP 100 and 101 planes, possibly because of the low resolution due to the binning of the wavevectors. However, we do see a peak that is not seen by Iacopini et. al. which may correspond to the FCC 002 peak, though better quality data is needed to confirm this.

\subsection{Velocity autocorrelation function}

Now that we have determined that the structure and phase behaviour match the experimental system, we use this model to measure some dynamical properties. For a particle with Brownian dynamics, it can be shown that the short time decay of the velocity autocorrelation function $C(t)$ should be exponential, while at long delay times $C(t)$ is expected to decay following a $t^{-3 / 2}$ power law. [37]

Both experiment $[19,21]$ and simulation [18] see this power law decay at long delay times, but for the experimental system $C(t)$ always changes sign and decays to zero from below, except at in extremely dilute samples, while the simulation results do not become negative until packing fractions get close to freezing.[18] The cause of this discrepancy could be that the single component HS system lacks the viscoelasticity of the solvent and the momentum transfer that occurs via the solvent.

Fig. 6 is a plot of the magnitude of the colloidal particle velocity autocorrelation function $|C(t)|$ from very low packing fractions up to just above freezing. For the lowest packing fraction (single colloidal particle in solvent) $C(t)$ decays exponentially initially (shown by a linear behaviour on a log-linear plot) but later changes into (roughly) a power law decay (shown by dotted line), which appears to be decaying to zero from above.

For the next largest packing fraction where more than one colloidal particle is present $(\eta=0.01$ corresponds to 10 colloidal particles) the velocity autocorrelation function changes sign (indicated by the spike downward in $|C(t)|)$ and then follows a power law decay from below zero. This change in behaviour could be due to the fact that having more than one colloidal particle present means they can interact (either directly or through momentum transfer via the solvent) and cause velocity reversals. $C(t)$ for all larger $\eta$ (except the extremely dilute case) become negative and decay to zero from below, but measurements at longer delay times for packing fractions of 0.18 , 


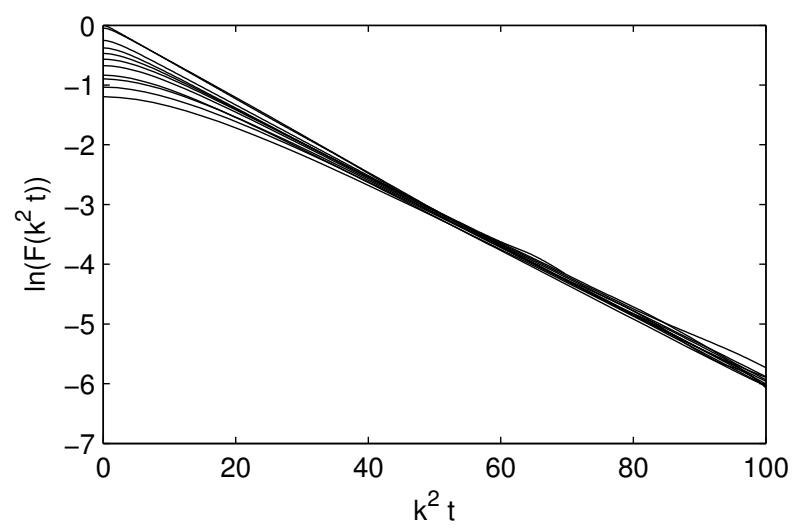

Figure 7. Plot of $\ln (F(k, t))$ vs. $k^{2} t$ measured in simulation at a packing fraction of 0.01 for the first 10 wavevectors given in Figure 1. These range of wavevectors extends from the smallest value measured $\left(k d_{1}=1.50\right)$ up to just past the structure factor peak $\left(k d_{1}=9.04\right)$. The data has been shifted down to show the agreement of the slope at long delay times.

0.27 and 0.36 will need to be done to determine whether we see the expected power law decay. We also see a big reduction in the delay time of the velocity reversals as the packing fraction gets close to freezing, in agreement with previous simulation results.[18]

\subsection{Intermediate scattering function}

The intermediate scattering function $F(k, t)$ (defined in Eq. 3) measures the correlations in time between the $k$ dependent number densities of the colloidal particles in the fluid. A thorough study of $F(k, t)$ over a large range of packing fractions is reserved for later work; in this work we look very briefly at the behaviour of $F(k, t)$ in a very dilute system using a very simple model. Since $F(k, t)$ describes the decay of fluctuations in the colloidal particle number density, we can invoke the Onsager regression hypothesis and state that the decay of the microscopic density is governed by the same equation as the macroscopic, that is;

$$
\frac{\partial}{\partial t} \delta n(k, t)=-k^{2} D_{0} \delta n(k, t)+R(k, t)
$$

where $\delta n(k, t)$ is a fluctuation in the $k$ dependent number density, $D_{0}$ is the dilute diffusion coefficient and $R(k, t)$ is the random component of the density fluctuations. This equation is just the spatial Fourier transform of the macroscopic diffusion equation with an additional random term. From this, we obtain a simple equation for the density autocorrelation function, which is easily solved giving

$$
F(k, t)=\frac{\left\langle n(k, t) n(k, 0)^{*}\right\rangle}{\left\langle|n(k, 0)|^{2}\right\rangle}=\exp \left(-k^{2} D_{0} t\right)
$$

This shows that for a dilute fluid where density fluctuations decay purely by diffusion $F(k, t)$ should decay exponentially. To compare this theoretical equation to simulation results, in Fig. 7 we have plotted $\ln (F(k, t))$ vs. $k^{2} t$ for the first 10 wavevectors at a packing fraction of 0.01 . These wavevectors correspond to the first 10 points shown in Fig. 1, covering a range of wavevectors from the lowest possible value measured in the simulation to just past the structure factor peak. Plotting the data in this way means according the Eq. 6, the line should be linear. Because it takes slightly longer to reach diffusive behaviour as the wavevector is increased, each line has been shifted down so that the behaviour at long delay times matches up. The top most line (which is unshifted) corresponds to the smallest wavevector measurements and thus to density oscillations the length of the simulation box. Its behaviour is diffusive (even at small delay times) shown by 
the linear trend. As the wavevector increases, the length of the density oscillation being probed is reduced, and it takes longer to approach diffusive behaviour. The long delay-time behaviour of $\ln (F(k, t))$ has linear decay for all wavevectors, with each having a similar gradient. This shows that for very dilute systems, density fluctuations decay via diffusion with a diffusion coefficient (given by the gradient of these lines) that is (almost) wavevector independent. This is in agreement with previous experimental work on light scattering of large spherical viruses [38] as well as dilute colloidal suspensions.[33]

It is expected that at larger $\eta$ the decay of $F(k, t)$ would become more complicated and Eq. 6 would have to be generalised to take into account a wavevector dependent diffusion coefficient, or even other processes besides diffusion which contribute to the density fluctuations. This will be the subject of future work.

\section{Conclusion}

In this work we constructed a model for a two component colloidal suspension that has structural properties matching experiment and theory for a HS system. In contrast to a single component HS system, the colloidal particles interact with an explicit solvent making their dynamics diffusive, rather than ballistic. We did this by modelling the colloidal particles and solvent using a WCA potential that is modified to include a hard-core. By using different values of the hard-core parameter in the pair potentials, we were able to create two species of particles with a size ratio of 4.034:1 and mass ratio of 50:1.

Different values were tested for the solute-solvent hard-core parameter in order to find the model that has structural properties which best match theoretical and experimental results. It was found that using the usual mixing rules to determine the value of the coefficient (taking an average of solute-solute and solvent-solvent interactions) caused large depletion effects. This is because of the excluded volume around each of the colloidal particles that the solvent cannot access. These depletion forces are not present in a real colloidal suspension as the size ratio is always much larger. We found that these effects can be effectively eliminated by reducing the hard-core parameter in the solute-solvent interactions and thus allow the solvent to exist closer to the colloidal particles.

In order to determine the best value for the hard-core in the solute-solvent interaction, a comparison was done between systems with three different values. System A used the usual mixing rules, system B reduced the parameter to allow the solvent centre of mass to reach the outside of the colloidal particles, and system $\mathrm{C}$ reduced the parameter to zero. The fluid static structure factor and radial distribution function was measured for each of the three systems and compared against the Percus-Yevick theory for hard-sphere systems which has been shown to closely match the structure of experimental colloidal suspensions. In doing so it was found that system $\mathrm{C}$ was the best match to the theoretical and experimental systems, and so this parameter was chosen for the rest of the work.

We then studied the phase behaviour by measuring the structure factor and radial distribution function at packing fractions corresponding to fluid, coexistence and crystal regions. The phase changes appeared to occur at the packing fractions expected for a one component HS system, indicating that the presence of the solvent has not changed the phase boundaries. The crystal static structure factor was measured by averaging over all $k$ vectors of equal magnitude that are consistent with the periodic boundary conditions. This allowed us to determine the positions of the peaks in the crystal structure factor which we compared to previous light scattering experiments. The positions of the peaks were found to be in good agreement showing that our system crystallises by stacking random hexagonal closed packed planes. The magnitude of the peaks did not match experiment. This is most likely because we are not able to average over all directions in the crystal.

Experimental and simulation results for the velocity autocorrelation function (VACF) have been compared previously. Both methods displayed the classic $-3 / 2$ power law decay at large 
delay times, but experimental findings show that it decays to zero from below (except for the extremely dilute systems), while in simulation it decays from above up to packing fractions close to freezing. Because of this discrepancy, we computed the VACF over a large range of packing fractions and found that our model also had the $-3 / 2$ power law decay, and that the VACF decayed to zero from above for the extremely dilute system, then decayed from below for all higher packing fractions, in agreement with experiment.

The intermediate scattering function was computed for a dilute system and compared against a simple model for its decay. We found that after an initial time-delay the correlation function decayed exponentially. This indicated that in a dilute fluid, long wavelength density fluctuations decay purely via diffusion with a wavevector independent diffusion coefficient, as we would expect. Later work will be aimed at studying the ISF at larger packing fractions in greater detail.

\section{Acknowledgements}

We wish to acknowledge the support of the Victorian Partnership for Advanced Computing (VPAC) for the use of their high-performance computing facilities. S.H. would also like to thank the Australian government for its support through the Australian Postgraduate Award. This paper is dedicated to the memory of Prof Ian Snook.

\section{References}

[1] Alder BJ, Wainwright TE. Studies in molecular dynamics. I. General method. J Chem Phys. 1959;31:459-466.

[2] Ramaswamy S. Issues in the statistical mechanics of steady sedimentation. Adv Phys. 2001;50:297-341.

[3] Pusey PN, van Megen W. Phase behaviour of concentrated suspensions of nearly hard colloidal spheres. Nature. 1986;320:340-342

[4] McPhie MG, Daivis PJ, Snook IK. Viscosity of a binary mixture: Approach to the hydrodynamic limit. Phys Rev E. 2006;74:031201.

[5] Hoover WJ, Ree FH. Melting transition and communal entropy for hard spheres. J Chem Phys. 1986;49:3609-3617.

[6] Ottewill RH, Richardson RA. Studies of particle-particle interactions using polystyrene lattices and time-average lightscattering. Coll Poly Sci. 1982;260:708-719.

[7] Robertus C, Philipse WH, Joosten JGH, Levine YK. Solution of the Percus-Yevick approximation of the multicomponent adhesive spheres system applied to the small-angle X-ray-scattering from microemulsions. J Chem Phys. 1989;90:44824490

[8] Duits MHG, May RP, Vrij A, Dekruif CG. Small-angle neutron-scattering of concentrated adhesive-hard-sphere dispersions. Langmuir. 1991;7:62-68.

[9] Frenkel D, Vos RJ, de Kruif CG, Vrij A. Structure factors of polydisperse systems of hard spheres: A comparison of Monte Carlo simulations and Percus-Yevick theory. J Chem Phys. 1986;84:4625-4630.

[10] Sun ZG, Tomlin CD, Sevick-Muraca EM. Investigation of particle interactions in concentrated colloidal suspensions using frequency domain photon migration: Monodisperse systems. J Col Int Sci. 2002;245:281-291.

[11] Schatzel K, Ackerson BJ. Density-fluctuations during crystallization of colloids. Phys Rev E. 1993;48:3766-3777.

[12] Harland JL, van Megen W. Crystallization kinetics of suspensions of hard colloidal spheres. Phys Rev E. 1997;55:30543067.

[13] Auer S, Frenkel D. Prediction of absolute crystal-nucleation rate in hard-sphere colloids. Nature. 2001;409:1020-1023.

[14] Filion L, Hermes M, Ni R, Dijkstra M. Crystal nucleation of hard spheres using molecular dynamics, umbrella sampling, and forward flux sampling: A comparison of simulation techniques. J Chem Phys. 2010;133:244115.

[15] Schilling T, Dorosz S, Schope HJ, Opletal G. Crystallization in suspensions of hard spheres: a Monte Carlo and molecular dynamics simulation study. J Phys Condens Mat. 2011;23:2194120.

[16] Radu M, Schilling T. Solvent hydrodynamics speed up crystal nucleation in suspensions of hard spheres. EPL. 2014;105:26001.

[17] Roehm D, Kesselheim S, Arnold A. Hydrodynamic interactions slow down crystallization of soft colloids. Soft Matter. 2014;10:5503-5509.

[18] Williams SR, Bryant G, Snook IK, van Megen W. Velocity autocorrelation functions of hard-sphere fluids: Long-time tails upon undercooling. Phys Rev Let. 2006;96:087801.

[19] van Megen W. Dynamical perspective of the freezing transition of a suspension of hard spheres from the velocity autocorrelation function. Phys Rev E. 2006;73:020503.

[20] van Megen W, Bryant G. Dynamical heterogeneity and the freezing transition in hard-sphere suspensions: Further analysis of the mean square displacement and the velocity autocorrelation function. Phys Rev E. 2007;76:0021402.

[21] Paul GL, Pusey PN. Observation of a long-time tail in Brownian motion. J Phys A: Math Gen. 1981;14:3301-3327.

[22] Smith ER, Snook IK, van Megen W. Hydrodynamic interactions in brownian dynamics I. Periodic boundary conditions for computer simulation. Physica. 1987;143A:441-467.

[23] Hernandez JP, de Pablo JJ, Graham D. Many-particle hydrodynamic and electrostatic interactions in a confined geometry. PRL. 2007;98:140602 
[24] Snook I, O’Malley B, McPhie M, Daivis P. The approach to the Brownian limit in particulate dispersions. J Mol Liq 2003;103:405-421.

[25] Vrabecz A, Toth G. Simulation of binary hard-sphere systems with 1:5 and 1:10 size ratios. Mol Phys. 2006;104:18431853.

[26] Asakura S, Oosawa F. Interactions between particles suspended in solutions of macromolecules. J Polym Sci. 1958;33:183-192.

27] Kihara T. Intermolecular Forces. Wiley: New York; 1976.

[28] Plimpton S. Fast Parallel Algorithms for Short-Range Molecular Dynamics. J Comp Phys. 1995;117:1-19.

[29] Hess S, Kroger M, Voigt H. Thermomechanical properties of the WCA-Lennard-Jones model system in its fluid and solid states. Physica A. 1998;250:58-82.

[30] McPhie M. Thermal and transport properties of shearing binary fluids [Ph.D]. Melbourne (Vic): RMIT University 2003.

[31] Iacopini S, Palberg T, Schope H. Crystallization kinetics of polydisperse hard-sphere-like microgel colloids: Ripening dominated crystal growth above melting. J Chem Phys. 2009;130:084502.

[32] Schope H, Bryant G, van Megen W. Effect of polydispersity on the crystallisation kinetics of suspensions of colloidal hard spheres when approaching the glass transition. J Chem Phys. 2007;127:084505.

[33] Martinez VA, Thijssen JHJ, Zontone F, van Megen W, Bryant G. Dynamics of hard sphere suspensions using dynamic light scattering and X-ray photon correlation spectroscopy: Dynamics and scaling of the intermediate scattering function. J Chem Phys. 2011;134:054505.

[34] van Megen W, Martinez VA, Bryant G. Arrest of flow and emergence of activated processes at the glass transition of a suspension of particles with hard spherelike interactions. Phys Rev Let. 2009;102:168301.

[35] Eckert T, Richtering W. Thermodynamic and hydrodynamic interaction in concentrated micro gel suspensions: Hard or soft sphere behavior?. J Chem Phys. 2008;129:124902.

[36] O'Malley B, Snook I. Crystal nucleation in the hard sphere systems. Phys Rev Let. 2003;90:085702.

[37] Hansen JP, McDonald I. Theory of simple liquids. Academic press: London; 1986.

[38] Pusey PN, Koppel DE, Schaefer DW, Camerini-Otero RD. Intensity fluctuation spectroscopy of laser light scattering by solutions of spherical viruses - R17, Q. $\beta$., BSV, PM2 and T7. 1. Light-scattering technique. Biochem. 1974;13:952-960. 